\title{
Repetitive transcranial magnetic stimulation: An innovative medical therapy
}

\author{
Georgios Mikellides, ${ }^{1}$ Panayiota Michael, ${ }^{2}$ Marianna Tantele ${ }^{3}$ \\ ${ }^{1}$ Faculty of Psychology and Neuroscience, Maastricht University, Netherlands \\ ${ }^{2}$ Cyprus rTMS Centre, Larnaka, Cyprus \\ ${ }^{3}$ Faculty of Medicine, University of Nicosia, Nicosia, Cyprus
}

ARTICLE HISTORY: Received 6 August 2020/Revised 7 October 2020/Published Online 26 March 2021

\begin{abstract}
Repetitive Transcranial Magnetic Stimulation (rTMS) is an innovative, non-invasive and well tolerated method that could be used as a treatment option for a variety of neuropsychiatric disorders. A large number of studies, for more than 30 years, have demonstrated that is a powerful neuroscience tool for diagnostic and therapeutic purposes. rTMS is based on the phenomenon of electromagnetic mutual induction, that firstly reported by Michael Faraday in 1831. Later, in 1985, Anthony Barker and his colleagues developed the first modern transcranial magnetic stimulation (TMS) device. rTMS uses brief electromagnetic pulses generated by an insulated coil, placed over the scalp. This technique has the ability to modulate the cortical activity of the brain. Daily rTMS stimulation for several weeks has been shown to be effective in reducing symptoms of many neuropsychiatric disorders. Moreover, studies have shown that dorsolateral prefrontal cortex (DLPFC) has a crucial role in improving cognitive performance and, as a result, is a commonly used target area for the treatment of many neuropsychiatric disorders such as depression. 2008 was a significant year for TMS history, as FDA approved for first time a TMS therapy device for the clinical treatment of depression. This approval as well as the National Institute for Health and Care Excellence (NICE) recommendation on TMS for the treatment of depression in 2015 lead to the establishment of rTMS as a first-line treatment for patients that failed in at least one prior antidepressant medication, followed by FDA approvals for the treatment of migraines and OCD. This paper aims to enhance further some of the clinical usefulness of rTMS.
\end{abstract}

KEYWORDS: rTMS, neuropsychiatry, depressive disorders, cognitive neuroscience, brain stimulation.

\section{Introduction}

Repetitive Transcranial Magnetic Stimulation (rTMS) is a non-invasive medical therapy that is used as a treatment option for a variety of neuropsychiatric disorders. rTMS is a brain stimulation technique based on the production of short magnetic fields using an insulated coil placed over specific areas of the scalp.' These magnetic fields are of the same type as those used in magnetic resonance imaging (MRI). Magnetic pulses produce a weak electrical current over a targeted region of the brain that activates neuronal circuits. ${ }^{1}$ This treatment has been shown to be a safe and well tolerated and can be effective in patients with depression ${ }^{2}$ or other neuropsychiatric disorders. Depending on the stimulation frequency, rTMS may reduce or increase cortical excitability of specific areas of the cerebral cortex. In most people, Low Frequency rTMS (LF-rTMS) $(\leq 1 \mathrm{~Hz}$ ) has been shown to decrease cortical excitability and be inhibitory and on the other hand, High Frequency rTMS (HF-rTMS) ( $\geq 5 \mathrm{~Hz}$ ) has been shown to increase cortical excitability and be excitatory. ${ }^{3}$

\section{The history of TMS}

The history of transcranial magnetic stimulation seems to begin in the early 1830 s. rTMS is based on the phenom- 
enon of electromagnetic mutual induction, that firstly reported by Michael Faraday ${ }^{4}$ in 1831 . According to this phenomenon, there is a mutual relation between electrical current and magnetic fields. Later in 1896, D'Arsonval ${ }^{5}$ reported flickers of lights (magnetophosphenes) after the placement of the coil. ${ }^{6}$ Similarly in 1910 , Silvanus P. Thompson ${ }^{7}$ reported faint flickering illumination after the application of the coil. Some years later, in 1982, Polson et $\mathrm{al}^{8}$ dealt with the stimulation of superficial peripheral nerves using time-varying magnetic fields. The first magnetic stimulator was developed in the Department of Medical Physics, Sheffield University, United Kingdom by Anthony Barker and colleagues ${ }^{9}$ in 1985. It was a pivotal moment in the history of transcranial magnetic stimulation since they used for first time the TMS technique in an awake man and they demonstrated the effect of magnetic stimulation on motor cortex of the human brain. They reported that magnetic pulses produced induction of weak electrical current in the human brain, without causing pain or discomfort. Also, they proved that magnetic stimulation excites peripheral nervous system and that after the placement of the coil on the scalp (over contralateral motor cortex), movements of the opposite hand were produced. ${ }^{10}$

\section{Dorsolateral prefrontal cortex}

Dorsolateral prefrontal cortex (DLPFC) is associated with many cognitive and behavioral processes like decision making, planning, reward processing, working memory. ${ }^{11,12}$ Moreover, is considered as a perfect target for stimulation during rTMS treatment, as is an important part of the following circuits: mesocorticolimbic dopamine circuits (which are associated with mechanisms of motivation and reward) and serotonergic circuits (which are associated with emotional regulation). ${ }^{12}$ Several studies reported the capacity of rTMS to alter the hypoactivity of DLPFC, after a successful treatment. ${ }^{13}$ Application of rTMS over the DLPFC has been shown to be an effective treatment for many neuropsychiatric disorders and has a crucial role in improving cognitive performance. For example, after HF-rTMS over the DLPFC brain area, patients with depression were shown to receive antidepressant effects. $^{13}$

\section{Neuroplasticity}

According to Cramer et al, neuroplasticity (brain plasticity or neural plasticity) can be defined as "the ability of the nervous system to respond to intrinsic or extrinsic stimuli by reorganizing its structure, function and connections".14 This capacity of the brain to change and adapt, may occurred as a result of the development process, learning, the environment, in response to disease or a therapy. ${ }^{14}$ These changes in brain structure and function may appear throughout the life of a person. The presence of neuropsychiatric disorders is associated with changes in the structure and function of the brain. Particularly, people with mental and addictive disorders may have abnormalities in their limbic, prefrontal, and frontostriatal neural circuits. These circuits are associated with emotion regulation, perception, social interaction, etc. ${ }^{14}$ Noninvasive therapies have the ability to alter brain activity in the cerebral cortex, which offers different treatment options of neuropsychiatric disorders. ${ }^{14,15}$ rTMS is a therapy that promotes neuroplasticity, as it has the ability to reduce or increase cortical excitability of a specific area of the brain in patients with neuropsychiatric disorders such as depression. ${ }^{13}$

\section{Safety}

Precautions

During rTMS treatment it is of the utmost importance to take precautions for patients with electronic or magnetic implants such as cochlear implants, drug pumps, pacemakers because magnetic fields may alter the function of these devices. ${ }^{16}$

The most serious side effect of transcranial magnetic stimulation, during the treatment, is seizure. However, the occurrence of seizures is characterized as rare.16 According to a survey conducted about the risk of seizures, the risk of seizures in people without risk factors is less than 1 in 60,000 sessions. ${ }^{17}$ Most of the seizures occurred in people with risk factors such as congenital epilepsies or anatomical/brain damages. ${ }^{17}$ Regarding the stimulation frequency, seizures were no more likely to occur in HF-rTMS than in LF-TMS or single/paired-pulse TMS. In addition, seizures were more likely to occur within the first TMS sessions. ${ }^{17}$ Therefore, precautions should be taken for patients with a history of seizures but also for people with increased risk factors. ${ }^{16,17}$

\section{Side effects}

rTMS is a treatment option in cases where medication has not helped the patient sufficiently as well as when the person does not want to receive medication for any reason (due to the side effects of medication, pregnancy, etc). Compared with standard medication, rTMS seems to cause fewer side effects. Specifically, standard medication may cause side effects such as weight gain, sexual disorders, gastrointestinal disorders, sedation, blurred vision or dry mouth..$^{18}$ In contrast, the most common side effects of rTMS are: headaches ( 5-23\%), discomfort at the site of stimulation ( 20-40\%) and twitching of facial muscles. One uncommon side effect of rTMS are seizures $(<0,1 \%){ }^{16,19}$

Rapid mechanical deformation of the TMS stimulation coil produces a broadband acoustic artifact that can ex- 
ceed $140 \mathrm{~dB}$, which in value exceeds the recommended safety levels for the auditory system. ${ }^{16}$ However, only a small number of individuals experienced a hearing problem. In order to avoid hearing-related problems during TMS treatment, a number of measures are suggested such as the use of hearing protection (earplugs). ${ }^{16}$

\section{rTMS vs ECT}

Many randomized studies examine the effectiveness of ECT (Electroconvulsive Therapy) compared to rTMS therapy. ECT is a convulsive therapy that requires anesthesia. In contrast with rTMS, which does not require anesthesia, patients that undergo ECT reported cognitive side effects and seizure induction..$^{20}$ rTMS therapy does not need to intentionally cause seizures, which is credited to the positives of the therapy in contrast with ECT. ${ }^{21}$ Eranti et al studied the equivalence of rTMS with ECT. ${ }^{22}$ At the end of the treatment, Hamilton Depression Rating Scale (HAM-D) in the ECT group were significantly lower that the rTMS group. However, no difference was reported between ECT and rTMS groups in HAM-D scores at the 6-month follow-up. They concluded that ECT is more effective than rTMS, especially in the short-term treatment of depression. A meta-analysis by Berlim et al has shown that ECT is more effective for the treatment of major depressive disorder (MDD) in contrast with HF-rTMS, although no difference reported on dropout rates. ${ }^{20}$ Finally, a systematic review and meta-analysis by Chen et al studied the effects of ECT, Bilateral rTMS (B-rTMS), Left prefrontal rTMS (L-rTMS) and Right prefrontal rTMS (R-rTMS) on patients with $M^{2} D^{23}$ and found that ECT was more effective, but least tolerated. Nevertheless, R-rTMS found to be the best tolerated treatment for patients with MDD and B-rTMS found to have the most beneficial balance in terms of efficacy and acceptability.

\section{rTMS and neuropsychiatric conditions}

In more recent years, studies have looked at the effectiveness of rTMS in treating a variety of neuropsychiatric conditions such as depression, schizophrenia, bipolar disorder, anxiety disorders, obsessive-compulsive disorder, addiction disorders, post-traumatic stress disorder, chronic pain, fibromyalgia and dementia.

\section{Major Depressive Disorder}

The most preferable and commonly used target area for the treatment of MDD is the DLPFC. Left DLPFC has been found to be hypoactive in depressed patients and this is associated with many depressive symptoms such as negative emotional bias, rumination, appetite changes and decreased energy level. HF-rTMS over the left DLPFC lead to activation of this brain area and produces antidepressant effects. Additionally, LF-rTMS over the right DLPFC leads to decrease local activity and as a result produces antidepressant effects. ${ }^{2,24}$ In addition, results of a recent study in MDD patients showed that HF-rTMS $(10 \mathrm{~Hz})$ over the left DLPFC has beneficial effects on psychomotor speed and cognitive control. ${ }^{25}$ It is noteworthy that rTMS is reported as a promising treatment option for patients that were not helped from antidepressant medication. ${ }^{26}$ Moreover, according to a recent case study, rTMS is a promising treatment for patients with treatment-resistant depression who have been previously try ECT and did not respond. ${ }^{27}$ However, these results cannot be generalized since they occur from an individual case study.

\section{Obsessive-compulsive disorder (OCD)}

rTMS found to be an effective method in reducing some of the obsessive-compulsive (OC) symptoms. A recent meta-analysis suggested that, active rTMS lead to significant reduction of Yale-Brown Obsessive Compulsive Scale (Y-BOCS) scores compared with sham rTMS. Also, they determined that application of LF-rTMS was more effective when applied over the supplementary motor area. However, application of active rTMS over the DLPFC produced benefit improvements of OC symptoms in contrast with sham rTMS.28

\section{Schizophrenia}

TMS demonstrated efficacy in decreasing negative symptoms and auditory hallucinations in schizophrenia. Schizophrenia patients that suffer from negative symptoms were found to have reduced activity in their prefrontal cortex (PFC). HF-rTMS over the PFC give rise in increasing local activation and causes significant improvements of the negative symptoms of schizophrenia. ${ }^{29}$

Kubera et al applied rTMS over superior temporal cortex (STC) in patients with auditory verbal hallucinations $(\mathrm{AVH}){ }^{30}$ This brain area is associated with increased cortical activity in patients who suffer from the positive symptoms of schizophrenia such as AVH. A meta-analysis of Zhang et al suggested that LF-rTMS over the left temporoparietal cortex may be an effective treatment for patients with auditory hallucinations. ${ }^{31}$ They noted reduction of severity of auditory hallucinations in patients with schizophrenia spectrum disorders after the application of the treatment. The combination of HF-rTMS over the left DLPFC with LF-rTMS over the Wernicke's area on the left temporoparietal cortex or over the right DLPFC was found to be effective in reducing negative symptoms, delusion and auditory hallucinations of schizophrenia patients. $^{32}$

\section{Addictions}

Barr et al reported that HF- rTMS over the DLPFC is a promising treatment option for addicted patients, as it 
decreases craving levels in patients who are addicted in tobacco, alcohol and cocaine. ${ }^{33}$ Specifically, regarding nicotine addiction, active HF-rTMS was found to decrease significantly the level of smoking craving, the number of cigarettes, cigarette consumption and nicotine dependence comparing with sham rTMS. In alcohol addiction, active HF-rTMS was found to decrease significantly the level of alcohol craving and alcohol consumption comparing with sham rTMS. Finally, in cocaine addiction, HF-rTMS was found to decrease the level of cocaine craving. DLPFC is the preferable rTMS target area for treating nicotine, alcohol and cocaine addiction, although for cocaine addiction is not sure yet whether rTMS over the right or left DLPFC is more effective.

According to a recent study, daily rTMS (MRI-guided) in the left DLPFC for 10 days may reduce cigarette consumption as well as the desire for up to a month and at the same time increases the likelihood of smoking cessation. ${ }^{34}$ In addition, rTMS appears to be a promising treatment for cocaine use disorder, as it appears to have a therapeutic role in reducing cocaine use and associated symptoms such as sleep disturbance and other adverse symptoms. ${ }^{35}$

\section{Bipolar Disorder}

rTMS is a promising treatment option for patients with both monopolar and bipolar depression. ${ }^{36}$ Patients with bipolar disorder (BD) reported significant cognitive improvement after the application of HF-rTMS over their left DLPFC. This cognitive improvement was associated with significant improvement in their working memory and processing speed, without causing them any adverse side effects. ${ }^{37}$

Although studies indicate that rTMS is a well-tolerant and safe treatment for $B D$, its efficacy results are mixed for episodes of mania and depression as well as for mixed state. ${ }^{38}$ These studies do not present an advantage of rTMS over sham stimulation. ${ }^{38}$ Additional randomized controlled trials are needed in the future in order to determine the effectiveness of $\mathrm{rTMS}$ for BD. ${ }^{38}$

\section{Anxiety \& Anxiety related disorders}

rTMS is considered to be a promising treatment for anxiety symptoms. It is presented as an effective and safe treatment for generalized anxiety disorder without serious side effects. ${ }^{39}$ Application of rTMS over the right DLPFC is an effective therapy for treating post-traumatic stress disorder (PTSD). ${ }^{40}$ Patients reported reduction of PTSD, anxiety and depressive symptoms after 10 daily sessions. ${ }^{11}$ LF-rTMS over the right DLPFC resulted in significant improvement of panic symptoms for patients with panic disorder. ${ }^{41}$

\section{Chronic Pain/Fibromyalgia}

rTMS is a promising treatment for neuropathic pain of various origins, such as central pain, pain from peripheral nerve disorders, fibromyalgia, and migraine..$^{42} \mathrm{~A}$ recent study showed that four consecutive HF- rTMS sessions (20 $\mathrm{Hz}$ ) every 3 weeks in the primary motor cortex have as a result a sustained analgesic effect. ${ }^{43}$

HF-rTMS over the primary motor cortex in patients with fibromyalgia produce positive long-lasting improvements in their quality of life and reduction of chronic pain. rTMS is an effective treatment for fibromyalgia patients without adverse effects on pain and mood levels. ${ }^{44,45}$

\section{Dementia/Alzheimer's Disease}

rTMS over the DLPFC improves behavioral and psychological symptoms of patients with dementia. ${ }^{46} \mathrm{~A}$ recent meta-analysis of randomized controlled trials have shown that rTMS is an effective treatment for cognitive impairment in patients with Alzheimer's disease. ${ }^{47}$ Patients with Alzheimer's disease reported significant improvement of their cognitive performance after the rTMS treatment. ${ }^{48}$ Bilateral DLPFC stimulation in combination with long term treatment proved to have the greater effectiveness in improving the cognitive ability of patients with Alzheimer's disease. ${ }^{49}$

\section{Improvement of Cognition}

Studies suggested that rTMS can be an effective and safe treatment for patients with mild cognitive impairment. ${ }^{48}$ Application of HF-rTMS over the DLPFC in older patients has been shown to benefit their cognition without any serious adverse effects. ${ }^{50}$ The stimulation of the DLPFC is associated with improvements in attention, verbal fluency, executive function and working memory. ${ }^{51} \mathrm{~A}$ meta-analysis of Chou et al showed that HF-rTMS over the left DPLFC and LF-rTMS over the right DLPFC is effective in improving memory functions. ${ }^{48}$

Finally, rTMS is a promising treatment option for some neurological disorders such as Parkinson's disease, ${ }^{52,53} \mathrm{mi}$ graines, ${ }^{54,55}$ as well as for recovery after a stroke. ${ }^{56,57}$

\section{Approved TMS treatments}

The Food and Drug Administration (FDA) approved TMS for depression, OCD and migraine. In October 2008, FDA approved Neuronetics NeuroStar TMS System as a treatment of MDD for adult patients that failed in at least one prior antidepressant medication. It was the first time that FDA approved a TMS device for the clinical treatment of depression (FDA approval K061053). FDA approval was based on study of O'Reardon et al in which 301 patients were divided into two groups (Active TMS group and Sham TMS group). ${ }^{58}$ Significant improvements of de- 
pressive symptoms in Active TMS group compared with Sham group were demonstrated. Patients reported mild adverse effects.

In December 2013, FDA approved the Cerena Transcranial Magnetic Stimulator (Cerena TMS) for treating migraine headaches preceded by an aura. Cerena TMS is a portable and hand-held device. This stimulation may stop or lessen migraines headaches with aura. FDA approval was based on a study in which 201 patients with moderate to strong migraine headaches divided into two groups (Cerena TMS group and Sham control group). Significant difference between the two groups regarding the effectiveness of the device was found. Specifically, the rate of patients in the Cerena TMS group who reported no pain 2 hours and 24 hours after the treatment were higher than the rate of the Sham control group. However, the study didn't support effectiveness in reducing other symptoms associated with migraine such as photophobia, phonophobia, nausea. Reported adverse effects were rare. ${ }^{59}$

In August 2018, FDA approved the Brainsway Deep TMS System for treating OCD. This FDA approval was based on a study in which 100 patients were divided into two groups (Brainsway Device group and Sham group). The results had shown that $38 \%$ of patients using Brainsway device reported reduction in YBOCS score compared with $11 \%$ of patients in the Sham group. Reported adverse effects were mild or moderate, except of headaches which were more frequent. ${ }^{60}$

The National Institute for Health and Care Excellence (NICE) in January 2014 approved TMS for the treatment and prevention of migraines ${ }^{61}$ and approximately one year later, in December 2015, approved TMS as an effective and safe treatment for depression. ${ }^{62}$

\section{Theta Burst Stimulation}

Theta Burst Stimulation (TBS) is one of the newer forms of rTMS protocols. TBS has the potential to cause long-term changes in cerebral cortex excitability in a much shorter stimulation period. Therefore, it has attracted research interest. ${ }^{63}$ Different types of TBS protocols have been developed and can be used during rTMS therapy. The main types of TBS protocols are the intermittent Theta Burst Stimulation (iTBS) which is shown to increase cortical excitability and the continuous Theta Burst Stimualtion (cTBS) which reduce cortical excitability. ${ }^{64}$ Additional types of TBS protocols that have been studied in recent years are accelerated $\mathrm{iTBS}^{65}$ intensive cerebellar iTBS (iCiTBS) ${ }^{66}$ and cerebel$\operatorname{lar}$ CTBS. ${ }^{63}$

According to a recent study by Blumberger et al in 2018, which compared the efficacy and safety of the standard depression protocol (lasting 30 minutes) with an iTBS pro- tocol (lasting 3 minutes), TBS found to have similar if not better effects on brain activity compared with the standard rTMS protocol. ${ }^{67}$ Based on this study, in August 2018 FDA approved MagVenture's TBS protocol for the treatment of depression.

\section{Conclusion}

Over the past decades, many clinical studies have demonstrated the effectiveness of brain stimulation in treating neuropsychiatric disorders. rTMS is a promising brain stimulation therapy based on the production of short magnetic fields using an insulated coil placed over the scalp and has the ability to change the cortical excitability. It is a very challenging field for clinical research in neuroscience as it helps depressed patients who have not been helped sufficiently from medication ${ }^{26}$ or patients who have not respond to ECT. ${ }^{27}$ In contrast with standard medication and ECT, rTMS causes fewer side effects and is a safe and well tolerated treatment. The approvals of FDA in 2008 and NICE in 2015 for depression indicated that rTMS can be used as a first line treatment for depressed patients that failed in at least one prior antidepressant medication.

It has been shown that rTMS is promising treatment option for many neuropsychiatric conditions like depression, ${ }^{2,24,25} \mathrm{OCD},{ }^{28} \mathrm{BD}^{36,37}$ negative symptoms ${ }^{29}$ and auditory hallucinations ${ }^{30,31}$ in schizophrenia, addictions, ${ }^{33-35}$ anxiety and anxiety related disorders, ${ }^{39-41}$ fibromyalgia, ${ }^{44,45}$ chronic pain, ${ }^{42,43}$ and dementia/Alzheimer's disease. ${ }^{46-49}$ It is also very important that many studies reported that rTMS causes significant improvement of cognition. ${ }^{48,50,51}$ Furthermore, rTMS it's an important neuroscience research tool as it helps in understanding brain connectivity. ${ }^{68}$

However, rTMS is not used enough in neuropsychiatric practice. Future research regarding rTMS may focus in studying the ability of maintenance TMS treatment in preventing relapses with the creation of personalized treatment of each patient individually. ${ }^{2}$ Moreover, the inclusion of neuronavigation in rTMS helps in the reduction of errors that may appear during the treatment. Neuronavigation is a novel method that includes an individual and exact image-guided navigation of the TMS coil for stimulating a specific brain area based on the brain anatomy and mapping of spatial brain areas for each patient individually. ${ }^{69,70}$ Therefore, additional well-designed randomized controlled trials in larger populations are necessary in order to generalize these results, to assess how the potential positive effects of this treatment are maintained over time, to understand better the pathology of neuropsychiatric disorders as well as to improve both therapeutic stimulation protocols and increase the effectiveness of the treatment. 


\section{References}

1. Mikellides G. RTMS (Repetitive Transcranial Magnetic Stimulation) could be used as a First Line Treatment for Depression. Glob J Intellect Dev Disabil 2018, 5:555655, doi:10.19080/GJIDD.2018.05.555655

2. Baeken C, Brem AK, Arns M, Brunoni AR, Filipčić I, Ganho-Ávila A et al. Repetitive transcranial magnetic stimulation treatment for depressive disorders: current knowledge and future directions. Curr Opin Psychiatry 2019, 32:409-415, doi: 10.1097/YCO.0000000000000533

3. Maeda F, Keenan JP, Tormos JM, Topka H, Pascual-Leone A. Interindividual variability of the modulatory effects of repetitive transcranial magnetic stimulation on cortical excitability. Exp Brain Res 2000, 133:425-430, doi:10.1007/s002210000432

4. Faraday F. Experimental research in electricity. Volume 1. Taylor and Francis, London, 1839

5. D' Arsonval. Dispositifs pour la mesure des courants alternatifs de toutes frequencies. CR Soc Biol (Paris) 1896, 3:450-451

6. Geddes LA. History of Magnetic Stimulation of the Nervous System. J Clin Neurophysiol 1991, 8:3-9, doi:10.1097/00004691-199101000-00003

7. Thompson SP. A Physiological Effect of an Alternating Magnetic Field. Proc R Soc Lond 1910, 82:396-398, doi:10.1098/rspb.1910.0032

8. Polson MJ, Barker AT, Freeston IL .Stimulation of nerve trunks with time-varying magnetic fields. Medical \& Biological engineering \& computing 1982, 20:243-244, doi:10.1007/BF02441362

9. Barker AT, Jalinousm R, Freeston IL. Non-invasive magnetic stimulation of human motor cortex. Lancet 1985, 1:1106-1107, doi: 10.1016/s01406736(85)92413-4

10. Barker AT. An introduction to the basic principles of magnetic nerve stimulation. J Clin Neurophysiol 1991, 8:26-37, doi: 10.1097/00004691199101000-00005

11. Berlim MT, Van den Eynde F. Repetitive Transcranial Magnetic Stimulation Over the Dorsolateral Prefrontal Cortex for Treating Posttraumatic Stress Disorder: An Exploratory Meta-Analysis of Randomized, DoubleBlind and Sham-Controlled Trials. Can J Psychiatry 2014, 59:487-496, doi: 10.1177/070674371405900905

12. Tik M, Hoffmann A, Sladky R, Tomova L, Hummer A, Navarro de Lara L et al. Towards understanding rTMS mechanism of action: Stimulation of the DLPFC Causes Network-specific Increase in Functional Connectivity. Neuroimage 2017, 162:289-296, doi:10.1016/j.neuroimage.2017.09.022

13. Kumar S, Singh S, Kumar N, Verma R. The Effects of Repetitive Transcranial Magnetic Stimulation at Dorsolateral Prefrontal Cortex in the Treatment of Migraine Comorbid with Depression: A Retrospective Open Study. Clin Psychopharmacol Neurosci 2018, 16:62-66, doi: 10.9758/cpn.2018.16.1.62

14. Cramer SC, Sur M, Dobkin BH, O'Brien C, Sanger TD, Trojanowski JQ et al. Harnessing neuroplasticity for clinical applications. Brain 2011, 134:15911609, doi:10.1093/brain/awr039

15. Gulyaeva NV. Molecular Mechanisms of Neuroplasticity: An Expanding Universe. Biochemistry (Mosc) 2017, 82:237-242, doi: 10.1134/S0006297917 030014

16. Rossi S, Hallett M, Rossini PM, Pascual-Leone A, Safety of TMS Consensus Group. Safety, ethical considerations, and application guidelines for the use of transcranial magnetic stimulation in clinical practice and research. Clin Neurophysiol 2009, 120:2008-2039, doi: 10.1016/j.clinph.2009.08.016

17. Lerner AJ, Wassermann EM, Tamir DI. Seizures from transcranial magnetic stimulation 2012-2016: Results of a survey of active laboratories and clinics. Clin Neurophysiol 2019, 130:1409-1416, doi:10.1016/j.clinph.2019.03.016

18. Khawam EA, Laurencic G, Malone DA Jr. Side effects of antidepressants: an overview. Cleve Clin J Med 2006, 73:351-361, doi:10.3949/ccjm.73.4.351

19. Dobek CE, Blumberger DM, Downar J, Daskalakis ZJ, Vila-Rodriguez F. Risk of seizures in transcranial magnetic stimulation: a clinical review to inform consent process focused on bupropion. Neuropsychiatr Dis Treat 2015, 11:2975-2987, doi: 10.2147/NDT.S91126

20. Berlim MT, Van den Eynde F, Daskalakis ZJ. Efficacy and acceptability of high frequency repetitive transcranial magnetic stimulation (rTMS) versus electroconvulsive therapy (ECT) for major depression: a system- atic review and meta-analysis of randomized trials. Depress Anxiety 2013 30:614-623, doi: 10.1002/da.22060

21. Micallef-Trigona B. Comparing the effects of repetitive transcranial magnetic stimulation and electroconvulsive therapy in the treatment of depression: a systematic review and meta-analysis. Depress Res Treat 2014, 2014:135049, doi:10.1155/2014/135049

22. Eranti S, Mogg A, Pluck G, Landau S, Purvis R, Brown RG et al. A Randomized, Controlled Trial With 6-Month Follow-Up of Repetitive Transcranial Magnetic Stimulation and Electroconvulsive Therapy for Severe Depression. Am J Psychiatry 2007, 164:73-81, doi:10.1176/ ajp.2007.164.1.73

23. Chen JJ, Zhao LB, Liu YY, Fan SH, Xie P. Comparative efficacy and acceptability of electroconvulsive therapy versus repetitive transcranial magnetic stimulation for major depression: A systematic review and multiple-treatments meta-analysis. Behav Brain Res 2017, 320:30-36, doi: 10.1016/j.bbr.2016.11.028.

24. Janicak PG, Dokucu ME. Transcranial magnetic stimulation for the treatment of major depression. Neuropsychiatr Dis Treat 2015, 11:1549-1560, doi: $10.2147 /$ NDT.S67477

25. Corlier J, Burnette E, Wilson AC, Lou JJ, Landeros A, Minzenberg MJ et al. Effect of repetitive transcranial magnetic stimulation (rTMS) treatment of major depressive disorder (MDD) on cognitive control. J Affect Disord 2020, 265:272-277, doi: 10.1016/j.jad.2020.01.068

26. Carpenter LL, Janicak PG, Aaronson ST, Boyadjis T, Brock DG, Cook IA et al. Transcranial magnetic stimulation (TMS) for major depression: a multisite, naturalistic, observational study of acute treatment outcomes in clinical practice. Depress Anxiety 2012, 29:587-596, doi: 10.1002/da.21969

27. Mikellides G, Tantele M. A Case of Treatment Resistant Depression Who Did Not Respond to ECT (Electroconvulsive Therapy) and Responded to rTMS (Repetitive Transcranial Magnetic Stimulation). Biomed J Sci \& Tech Res 2018, 8, doi: 10.26717/BJSTR.2018.08.001582

28. Rehn S, Elsick GD, Brakoulias V. A Meta-Analysis of the Effectiveness of Different Cortical Targets Used in Repetitive Transcranial Magnetic Stimulation (rTMS) for the Treatment of Obsessive Compulsive Disorder (OCD). Psychiatr Q 2018, 89:645-665, doi:10.1007/s11126-018-9566-7

29. Linsambarth S, Jeria A, Avirame K, Todder D, Riquelme R, Stehberg J. Deep Transcranial Magnetic Stimulation for the Treatment of Negative Symptoms in Schizophrenia: Beyond an Antidepressant Effect. J ECT 2019, 35:e46-e54, doi: 10.1097/YCT.00000000000000592

30. Kubera KM, Barth A, Hirjak D, Thomann PA, Wolf RC. Noninvasive brain stimulation for the treatment of auditory verbal hallucinations in schizophrenia: methods, effects and challenges. Front Syst Neurosci 2015, 9:131, doi: 10.3389/fnsys.2015.00131

31. Zhang Y, Liang W, Yang S, Dai P, Shen L, Wang C. Repetitive transcranial magnetic stimulation for hallucination in schizophrenia spectrum disorders: A meta-analysis. Neural Regen Res 2013, 8:2666-2676, doi: 10.3969/j. issn.1673-5374.2013.28.009

32. Mikellides G, Tantele M. rTMS for Treatment Resistant Schizophrenia. EC Psychology Psychiatry 2020, 9:1-4, doi: 10.31080/ecpp.2020.09.00656

33. Barr MS, Farzan F, Wing VC, George TP, Fitzgerald PB, Daskalakis ZJ. Repetitive transcranial magnetic stimulation and drug addiction. Int Rev Psychiatry 2011, 23:454-466, doi:10.3109/09540261.2011.618827

34. Li X, Hartwell KJ, Henderson S, Badran BW, Brady KT, George MS. Two weeks of image-guided left dorsolateral prefrontal cortex repetitive transcranial magnetic stimulation improves smoking cessation: A double-blind, sham-controlled, randomized clinical trial. Brain Stimul 2020, 13:1271-1279, doi: 10.1016/j.brs.2020.06.007

35. Gómez Pérez LJ, Cardullo S, Cellini N, Sarlo M, Monteanni T, Bonci A et al. Sleep quality improves during treatment with repetitive transcranial magnetic stimulation (rTMS) in patients with cocaine use disorder: a retrospective observational study. BMC Psychiatry 2020, 20:153, doi: 10.1186/ s12888-020-02568-2

36. Phillips AL, Burr RL, Dunner DL. Repetitive Transcranial Magnetic Stimulation in the Treatment of Bipolar Depression: Experience From a Clinical Setting. J Psychiatr Pract 2020, 26:37-45, doi: 10.1097/ PRA.0000000000000447 
37. Yang LL, Zhao D, Kong LL, Sun YQ, Wang ZY, Gao YY et al. High-frequency repetitive transcranial magnetic stimulation (rTMS) improves neurocognitive function in bipolar disorder. J Affect Disord 2019, 246:851-856, doi: 10.1016/j.jad.2018.12.102

38. Kozel FA. Clinical Repetitive Transcranial Magnetic Stimulation for Posttraumatic Stress Disorder, Generalized Anxiety Disorder, and Bipolar Disorder. Psychiatr Clin North Am 2018, 41:433-446, doi: 10.1016/ j.psc.2018.04.007

39. Cui H, Jiang L, Wei Y, Li W, Li H, Zhu J et al. Efficacy and safety of repetitive transcranial magnetic stimulation for generalized anxiety disorder: A meta-analysis. Gen Psychiatr 2019, 32:e100051, doi: 10.1136/ gpsych-2019-100051

40. Kan RLD, Zhang BBB, Zhang JJQ, Kranz GS. Non-invasive brain stimulation for posttraumatic stress disorder: a systematic review and meta-analysis. Transl Psychiatry 2020, 10:168, doi:10.1038/s41398-020-0851-5

41. Mantovani A, Lisanby SH, Pieraccini F, Ulivelli M, Castrogiovanni P, Rossi S. Repetitive Transcranial Magnetic Stimulation (rTMS) in the treatment of Panic Disorder (PD) with comorbid major depression. J Affect Disord 2007, 102:277-280, doi:10.1016/j.jad.2006.11.027

42. Yang S, Chang MC. Effect of Repetitive Transcranial Magnetic Stimulation on Pain Management: A Systematic Narrative Review. Front Neurol 2020, 11:114, doi: 10.3389/fneur.2020.00114

43. Quesada C, Pommier B, Fauchon C, Bradley C, Créac'h C, Murat M et al. New procedure of high-frequency repetitive transcranial magnetic stimulation for central neuropathic pain: a placebo-controlled randomized crossover study. Pain 2020, 161:718-728, doi:10.1097/j.pain. 0000000000001760

44. Boyer L, Dousset A, Roussel P, Dossetto N, Cammilleri S, Piano V et al. rTMS in fibromyalgia: a randomized trial evaluating $\mathrm{QoL}$ and its brain metabolic substrate. Neurology 2014, 82:1231-1238, doi:10.1212/ WNL.0000000000000280

45. Passard A, Attal N, Benadhira R, Brasseur L, Saba G, Sichere P et al. Effects of unilateral repetitive transcranial magnetic stimulation of the motor cortex on chronic widespread pain in fibromyalgia. Brain 2007, 130:26612670, doi: 10.1093/brain/awm189

46. Wang X, Mao Z, Yu X. The role of noninvasive brain stimulation for behavioral and psychological symptoms of dementia: a systematic review and meta-analysis. Neurol Sci 2020, 41:1063-1074, doi: 10.1007/ s10072-020-04245-4

47. Wang X, Mao Z, Ling Z, Yu X. Repetitive transcranial magnetic stimulation for cognitive impairment in Alzheimer's disease: a meta-analysis of randomized controlled trials. J Neurol 2020, 267:791-801, doi: 10.1007/ s00415-019-09644-y

48. Chou YH, Ton That V, Sundman M. A Systematic Review and MetaAnalysis of rTMS Effects on Cognitive Enhancement in Mild Cognitive Impairment and Alzheimer's disease. Neurobiol Aging 2020, 86:1-10, doi: 10.1016/j.neurobiolaging.2019.08.020

49. Lin Y, Jiang WJ, Shan PY, Lu M, Wang T, Li RH et al. The role of repetitive transcranial magnetic stimulation (rTMS) in the treatment of cognitive impairment in patients with Alzheimer's disease: A systematic review and meta-analysis. J Neurol Sci 2019, 398:184-191, doi:10.1016/j.jns.2019.01.038

50. Cheng CPW, Wong CSM, Lee KK, Chan APK, Yeung JWF, Chan WC. Effects of repetitive transcranial magnetic stimulation on improvement of cognition in elderly patients with cognitive impairment: a systematic review and meta-analysis. Int J Geriatr Psychiatry 2018, 33:e1-e13, doi: 10.1002/ gps. 4726

51. Serafini G, Pompili M, Belvederi Murri M, Respino M, Ghio L, Girardi P et al. The Effects of Repetitive Transcranial Magnetic Stimulation on Cognitive Performance in Treatment-Resistant Depression. A Systematic Review. Neuropsychobiology 2015, 71:125-139, doi: 10.1159/000381351

52. Lefaucheur JP, Drouot $X$, Von Raison F, Ménard-Lefaucheur I, Cesaro $P$, Nguyen JP. Improvement of motor performance and modulation of cortical excitability by repetitive transcranial magnetic stimulation of the motor cortex in Parkinson's disease. Clin Neurophysiol 2004, 115:25302541, doi: 10.1016/j.clinph.2004.05.025
53. Yang C, Guo Z, Peng H, Xing G, Chen H, McClure MA et al. Repetitive transcranial magnetic stimulation therapy for motor recovery in Parkinson's disease: A Meta-analysis. Brain Behav 2018, 8:e01132, doi: 10.1002/ brb3.1132

54. Misra UK, Kalita J, Bhoi SK. High frequency repetitive transcranial magnetic stimulation (rTMS) is effective in migraine prophylaxis: an open labeled study. Neurol Res 2012, 34:547-551, doi: 10.1179/1743132812Y.0000000045

55. Barker AT, Shields K. Transcranial Magnetic Stimulation: Basic Principles and Clinical Applications in Migraine. Headache 2017, 57:517-524, doi: 10.1111/head.13002

56. Fisicaro F, Lanza G, Grasso AA, Pennisi G, Bella R, Paulus W et al. Repetitive transcranial magnetic stimulation in stroke rehabilitation: review of the current evidence and pitfalls. Ther Adv Neurol Disord 2019, 12:1756286419878317, doi: 10.1177/1756286419878317

57. Ren $\mathrm{CL}$, Zhang GF, Xia N, Jin CH, Zhang XH, Hao JF et al. Effect of LowFrequency rTMS on Aphasia in Stroke Patients: A Meta-Analysis of Randomized Controlled Trials. PLoS One 2014, 9:e102557, doi: 10.1371/ journal.pone.0102557

58. O'Reardon JP, Solvason HB, Janicak PG, Sampson S, Isenberg KE, Nahas Z et al. Efficacy and safety of transcranial magnetic stimulation in the acute treatment of major depression: a multisite randomized controlled trial. Biol Psychiatry 2007, 62:1208-1216, doi:10.1016/j.biopsych. 2007.01.018

59. US Food and Drug Administration. De Novo classification request for cerena transcranial magnetic stimulator (TMS) device (Cited 5 March 2013). Available from https://www.accessdata.fda.gov/cdrh_docs/reviews/ K130556.pdf

60. US Food and Drug Administration. FDA permits marketing of transcranial magnetic stimulation for treatment of obsessive compulsive disorder (Cited 14 August 2018). Available from https://www.fda.gov/news-events/ press-announcements/fda-permits-marketing-transcranial-magnetic-stimulation-treatment-obsessive-compulsive-disorder

61. National Institute for Health and Care Excellence (NICE). Transcranial magnetic stimulation for treating and preventing migraine (Cited 23 January 2014) Available from https://www.nice.org.uk/guidance/ipg477

62. National Institute for Health and Care Excellence (NICE). Repetitive transcranial magnetic stimulation for Depression (Cited 16 December 2015). Available from https://www.nice.org.uk/guidance/ipg542

63. Sanna A, Follesa P, Puligheddu M, Cannas A, Serra M, Pisu MG et al. Cerebellar continuous theta burst stimulation reduces levodopa-induced dyskinesias and decreases serum BDNF levels. Neurosci Lett 2020, 716:134653, doi: 10.1016/j.neulet.2019.134653

64. Bradnam LV, McDonnell MN, Ridding MC. Cerebellar Intermittent ThetaBurst Stimulation and Motor Control Training in Individuals with Cervical Dystonia. Brain Sci 2016, 6:56, doi:10.3390/brainsci6040056

65. Duprat R, Desmyter S, Rudi de R, van Heeringen K, Van den Abbeele D, Tandt $\mathrm{H}$ et al. Accelerated intermittent theta burst stimulation treatment in medication-resistant major depression: A fast road to remission? Affect Disord 2016, 200:6-14, doi: 10.1016/j.jad.2016.04.015

66. Chauhan P, Garg S, Tikka SK, Khattri S. Efficacy of Intensive Cerebellar Intermittent Theta Burst Stimulation (iCiTBS) in Treatment-Resistant Schizophrenia: a Randomized Placebo-Controlled Study. Cerebellum 2020, 22:1-8, doi:10.1007/s12311-020-01193-9

67. Blumberger DM, Vila-Rodriguez F, Thorpe KE, Feffer K, Noda Y, Giacobbe $P$ et al. Effectiveness of theta burst versus high-frequency repetitive transcranial magnetic stimulation in patients with depression (THREE-D): a randomised non-inferiority trial. Lancet 2018, 391:1683-1692, doi: 10.1016/ S0140-6736(18)30295-2

68. Fox MD, Halko MA, Eldaief MC, Pascual-Leone A. Measuring and manipulating brain connectivity with resting state functional connectivity magnetic resonance imaging (fcMRI) and transcranial magnetic stimulation (TMS). Neuroimage 2012, 62:2232-2243, doi: 10.1016/j.neuroimage.2012.03.035

69. Remanan R, Panchal J, Lange D. Role of Neuronavigation for Training and Improvement of Accuracy During Transcranial Magnetic Stimulation. Neurology 2016, 86(16 Supplement)

70. Ruohonen J, Karhu J. Navigated transcranial magnetic stimulation. Neurophysiol Clin 2010, 40:7-17, doi: 10.1016/j.neucli.2010.01.006 


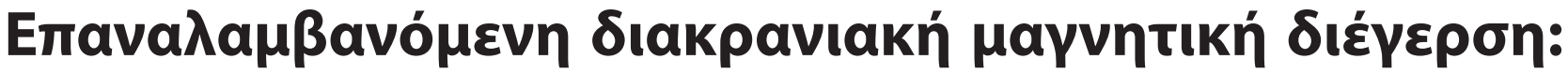

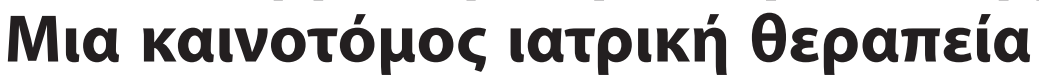

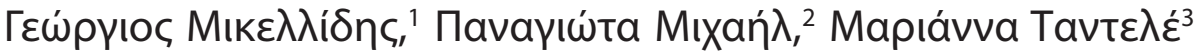

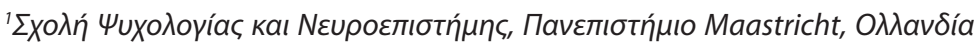

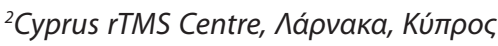

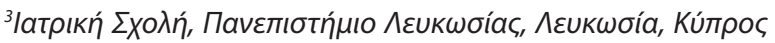

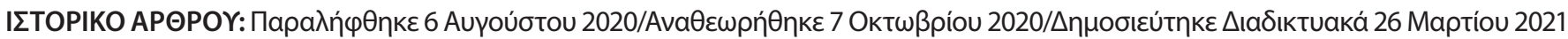

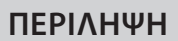

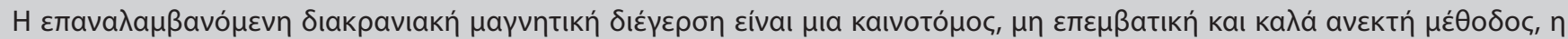

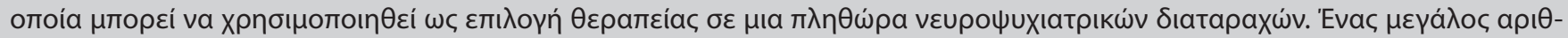

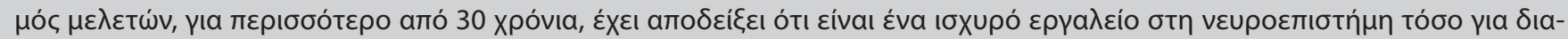

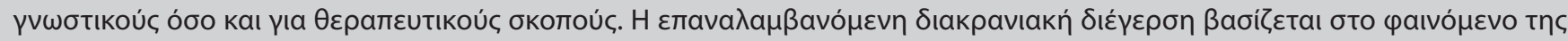

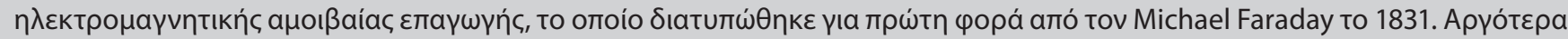

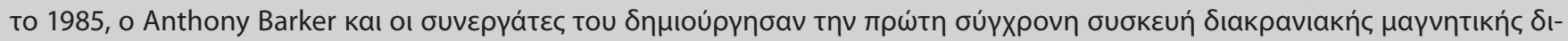

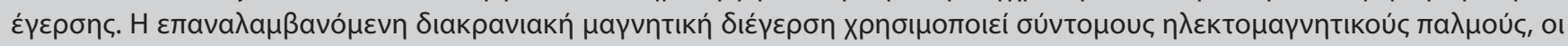

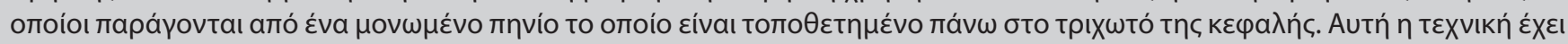

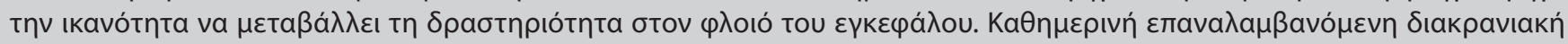

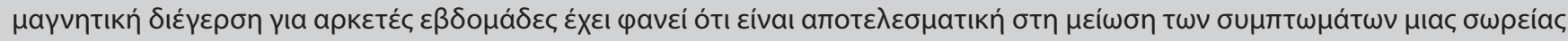

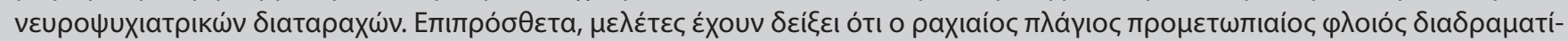

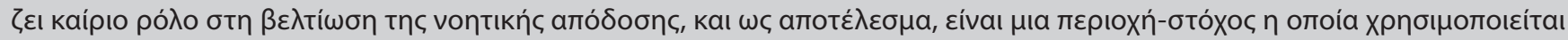

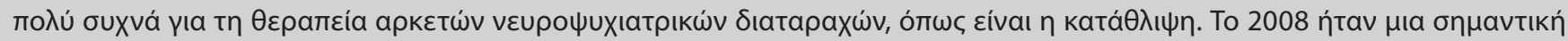

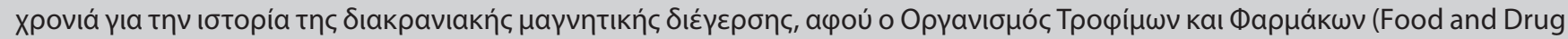

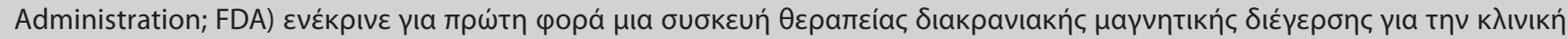
Ө

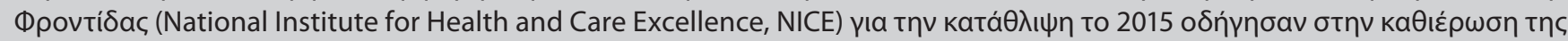

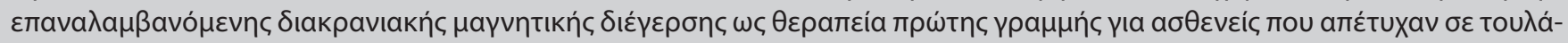

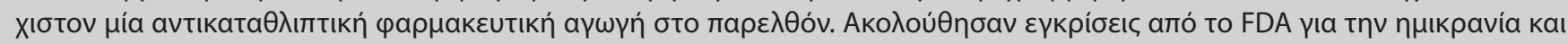

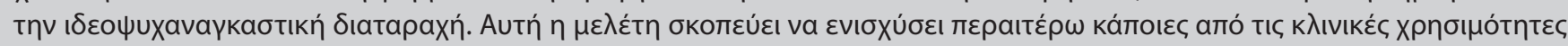

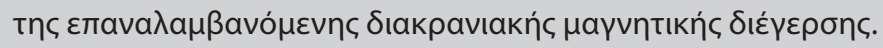

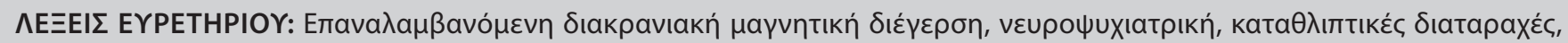

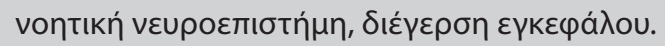

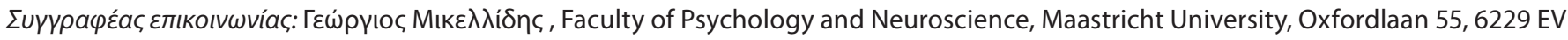

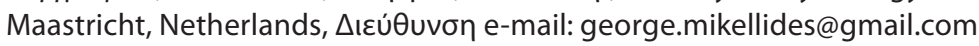

